MATEC Web of Conferences 11,01045 (2014)

DOI: $10.1051 /$ matecconf $/ 20141101045$

(C) Owned by the authors, published by EDP Sciences, 2014

\title{
Etude comparative de la cinétique de la réaction d'hydratation des bétons autoplaçants et des bétons vibrés
}

\author{
Gargouri Ahmed $^{1,2}$; Daoud Atef ${ }^{1,3}$; Kallel Abderrazak ${ }^{1}$; Loulizi Amara $^{4}$; , Makni Moncef ${ }^{1,2}$; Diouri Abdeljabbar ${ }^{5}$
}

1. Université de Tunis El Manar, Ecole Nationale d'ingénieurs de Tunis, Laboratoire Génie Civil, LR03ES05, 1002, Tunis, Tunisie ;

2. Institut supérieur des études technologiques de Sfax, 3099, Sfax, Tunisie ;

3. Université de Gabes, Ecole Nationale d'ingénieurs de Gabes, 6029, Gabes, Tunisie ;

4. Université de Tunis El Manar, Ecole Nationale d'ingénieurs de Tunis, Laboratoires des Matériaux, Optimisation et Energie pour la Durabilité, LR-11-ES16, 1002, Tunis, Tunisie

5. Laboratoire de Chimie du Solide Appliquée, Faculté des Sciences, Université MohammedV-Agdal, BP1014- Rabat, Maroc

Résumé :Les bétons autoplaçants (BAP) sont des bétons très fluides, absolument homogènes, mis en place sans vibration externe ou interne et présentant des résistances analogues à celles des bétons vibrés, ordinaires et à hautes performances (gamme de 25-80 MPa).De telles caractéristiques sont directement liées à leurs composition, qui en plus du ciment, de l'eau et des granulats (sable et gravillons) renferment des fines, un fluidifiant et assez souvent un agent de viscosité. Ces bétons présentent deux intérêts :

Le premier économique par la simplification de la mise en œuvre, l'amélioration de la sécurité et la réduction des nuisances sur et autour du chantier ;

Le second est technique par l'amélioration de la qualité et coulage de zones fortement armées.

Les BAP sont des solutions bien adaptées à des nombreuses applications, notamment des ouvrages telles que les dallages, les parois, les radiers, les planchers etc. Ces ouvrages généralement considérés comme ouvrages massifs sont soumis à plusieurs types de déformations pendant le processus d'hydratation du béton.

En effet, la nature exothermique de la réaction chimique du ciment peut induire des déformations de dilatation et de contraction. Par ailleurs, la dépression capillaire crée par la consommation d'eau due à l'hydratation du ciment entraine un retrait de dessiccation. Ces déformations peuvent entrainer des micros fissurations pouvant affecter la durabilité de l'ouvrage à long terme surtout pour les ouvrages épais. D'où l'importance d'étudier la cinétique d'hydratation de ses bétons non conventionnels et de les comparer à celle des bétons vibrés traditionnels. L'évolution de la température adiabatique ainsi que la variation en fonction du temps du degré d'hydratation sont déterminées pour le béton autoplaçant et le béton vibré. L'analyse des résultats expérimentaux obtenus montre que le changement de composition modifie considérablement la cinétique de la réaction d'hydratation.

\section{Introduction}

Le développement technologique dans la fabrication des bétons par la création de nouveaux additifs, à permit de formuler des bétons plus sophistiqués notamment le béton autoplacant. Ce matériau est caractérisé par sa fluidité, sa facilité de mise en œuvre et sa capacité à remplir les coffrages fortement armés.

La possibilité de concevoir un béton autoplaçant avec des granulats locaux parait particulièrement intéressant dans la mesure où ce matériau réuni des propriétés rhéologiques tel que la fluidité, absence de ségrégation et mécaniques comparable à celles d'un béton vibré conventionnel.
Les applications possibles de ce matériau sont nombreuse à savoir les chaussées, les dallages, les parois etc [4] ...Ces ouvrages généralement considérer comme ouvrages massifs sont soumis à plusieurs types de déformations pendant le processus d'hydratation du béton. En effet, la nature exothermique de la réaction chimique du ciment peut induire des déformations de dilatation et de contraction. Par ailleurs, la dépression capillaire crée par la consommation d'eau due à l'hydratation du ciment entraine un retrait de dessiccation. Ces déformations peuvent entrainer des micro fissurations pouvant affecter la durabilité de l'ouvrage à long terme surtout pour les ouvrages épais. Ces fissures sont appelées fissures de jeune âge pouvant se refermer totalement ou partiellement. D'où 
l'importance d'étudier la cinétique d'hydratation de ce béton non conventionnel et de le comparé à un béton vibré.

Dans ce papier on s'intéresse à la formulation d'un béton autoplaçant utilisant des matériaux locaux et du béton vibré. La cinétique de la réaction hydratation du béton autoplaçant retenu à base de granulats locaux est comparée à celle d'un béton vibré de référence. L'évolution de la température adiabatique ainsi que la variation en fonction du temps du degré d'hydratation sont déterminées et interprétées.

\section{Matériaux utilisés}

Les matériaux utilisés dans ce travail sont des matériaux locaux à savoir :

- Comme liant, nous avons utilisé un ciment Portland de type CEMI 42.5 N conforme à la norme NT 47.01. Pour limiter le dosage en ciment, sachant que le volume de pâte requis dans un BAP est toujours plus important en comparaison avec un béton classique vibré, nous avons utilisé un complément en filler calcaire $(\mathrm{CaCO} 3>99 \%)$ comme additif minérale.

- Un sable local (0-4 mm) et un gravier en deux coupures sachant $(4-8 \mathrm{~mm})$ et $(8-16 \mathrm{~mm})$ calcaire concassé ont aussi été utilisés comme granulats. Ils ont une densité de 2,$608 ; 2.737$ et 2.76 respectivement.

Pour atteindre les propriétés d'un BAP à l'état frais, et suite à une campagne d'essais, l'adjuvant retenu est celui un superplastifiant / haut réducteur d'eau polyvalent de nouvelle génération non chloré à base de copolymère acrylique contenant un agent colloïdale permet la fabrication de bétons plastiques à autoplaçants transportés sur de longues distances ,pompés et améliore la stabilité, limite la ségrégation du béton et rend les formules moins susceptibles aux variations d'eau et des constituants.

\section{Formulation des BAP}

Les méthodes de formulation des BAP sont très variées dont la plupart des formules restent empiriques. Ainsi un grand nombre de formulations est basé sur l'expérience acquise ces dernières années.[ROL 98,DES 05 et DES 07]. L'association française de génie civil a mis en place des techniques pour valider et caractériser à l'état frais les bétons autoplaçants, ce pendant, il n'existe pas de formulation et des méthodes généralisées comme pour le béton vibré. La méthode adoptée se base sur le remplissage des vides inter-granulaires par la pâte liante garantissant un comportement rhéologique autoplaçant des bétons. Le rapport $(\mathrm{G} / \mathrm{S})$ est égal environ à 1 , le dosage de ciment est de $\mathrm{C}=350 \mathrm{~kg} / \mathrm{m} 3$.

L'empilement optimal du sable et du gravier 4/8, déterminé par le Modèle d'Empilement Compressible en utilisant la méthode de la densité. Les quantités de superplastifiant ont été variées de façon à garantir les propriétés rhéologiques d'un béton autoplaçant.

Suite à une compagne des essais d'ouvrabilité, de mobilité en milieu confiné et non confiné, le BAP retenu, est celui présenté dans le tableau ci-dessous (Tableau1) :
Tableau 1 : Formulation du BAP

\begin{tabular}{|l|l|l|}
\hline \multicolumn{1}{|c|}{ formulation } & \multicolumn{1}{c|}{ Unités } & \multicolumn{1}{c|}{1} \\
\hline ciment & $\mathrm{kg} / \mathrm{m}^{3}$ & 350 \\
\hline gravier $4 / 8$ & $\mathrm{~kg} / \mathrm{m}^{3}$ & 540 \\
\hline gravier $8 / 16$ & $\mathrm{~kg} / \mathrm{m}^{3}$ & 360 \\
\hline sable $0 / 4$ & $\mathrm{~kg} / \mathrm{m}^{3}$ & 900 \\
\hline filaire & $\mathrm{kg} / \mathrm{m}^{3}$ & 100 \\
\hline eau & $1 / \mathrm{m}^{3}$ & 194,25 \\
\hline adjuvant & $\mathrm{kg} / \mathrm{m}^{3}$ & 7,7 \\
\hline
\end{tabular}

Les essais de caractérisation à l'état frais réalisés sont l'étalement au cône d'Abrams fig. (1a); la boite en L fig. (1b) et la stabilité au tamis fig. (1c).

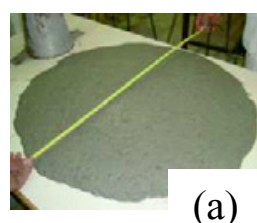

(a)

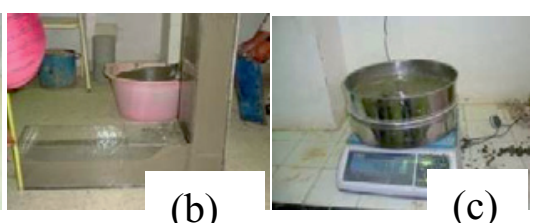

(c)
Fig(1) Essais de caractérisation à l'état frais : (a) étalement au cône d'Abrams ;(b) Boite en L ;(c) stabilité au tamis

Les résultats des essais à l'état frais sont récapitulés dans le tableau ci-dessous.

Tableau 2 : Valeurs des essais rhéologiques

\begin{tabular}{|l|l|l|}
\hline étalement & $(\mathrm{cm})$ & 67 \\
\hline boite en L & - & 0,8 \\
\hline stabilité au tamis & $\%$ & 4,88 \\
\hline air occlue & $\%$ & 1,8 \\
\hline masse volumique & $\mathrm{kg} / \mathrm{m}^{3}$ & 2402 \\
\hline
\end{tabular}

\section{Formulation du béton vibré}

- La formulation d'un béton de référence a été établie suivant la norme EN 480.1.1997. Les composants locaux de ce béton sont calculés et présentés dans le tableau ci dessous.

Tableau 3 : Composition du béton vibré testé

\begin{tabular}{|l|l|l|}
\hline Désignations & Unités & B. réf. \\
\hline Granulat calcaire concassé $(8 / 16)$ & $\mathrm{kg} / \mathrm{m}^{3}$ & 918.16 \\
\hline Granulat calcaire concassé $(4 / 8)$ & $\mathrm{kg} / \mathrm{m}^{3}$ & 229.54 \\
\hline Sable lavé de rivière $(0 / 4)$ & $\mathrm{kg} / \mathrm{m}^{3}$ & 730 \\
\hline Ciment CPA 45 & $\mathrm{kg} / \mathrm{m}^{3}$ & 350 \\
\hline Plastifiant & $1 / \mathrm{m}^{3}$ & 3.50 \\
\hline Eau & $1 / \mathrm{m}^{3}$ & 175 \\
\hline Masse théorique pour $1 \mathrm{~m}^{3}$ de béton & $\mathrm{kg} / \mathrm{m}^{3}$ & 2406.2 \\
\hline
\end{tabular}

Des granulats calcaires concassés de la carrière FAIDH ont été utilisés. Du sable lavé de propreté supérieure à $80 \%$, le ciment est du CEM I 42,5. Pour faciliter la mise en œuvre du matériau, un plastifiant à base de mélamine formol sulfoné a été utilisé dans le mélange. A fin d'éliminer les effets secondaires de cet adjuvant sur les propriétés du béton, on l'a utilisé aussi dans toutes les compositions pour valider le béton de référence.

Dans un objectif d'obtenir un béton ouvrable, on a opté à ce que l'affaissement sera environ de $10 \mathrm{~cm}$. 


\section{Etude de la cinétique de la réaction d'hydratation}

Le ciment portland est un mélange de clinker et de gypse dans lequel le clinker est formé par du calcaire et de l'argile à une température élevée. Ces composantes sont hydrauliques, ils présentent en présence d'eau des hydrates qui précipitent et se rassemble en une structure mécaniquement résistante.[10-12]. Les principaux constituants du ciment sont: Le silicate tricalcique $\mathrm{Ca}_{3} \mathrm{SiO}_{5}$, Le silicate bicalcique $\mathrm{Ca}_{2} \mathrm{SiO}_{4}$, l'Aluminate tricalcique $\mathrm{Ca}_{3} \mathrm{Al}_{2} \mathrm{O}_{6}$ et l'aluminoferrite bicalcique $\mathrm{Ca}_{2} \mathrm{AlFeO}_{5}$

Les mécanismes de l'hydratation du ciment portland sont multiples tels que l'adsorption, l'hydrolyse, la dissolution, la solvatation et la cristallisation ne sont toujours pas complètement élucidés. En effet, les divergences ne concerne que l'hydratation précoce et principalement du $\mathrm{C} 3 \mathrm{~S}$, puisque maintenant il est admis que le $\mathrm{C}_{3} \mathrm{~A}$ s'hydrate suivant un mécanisme de dissolution [12].

Les particules du système dispersé que forme la pate de ciment sont des grains polycristallins constitués de silicates et d'aluminates. Les composants minéraux du ciment présentent des vitesses d'hydratations différentes. Leurs réactions d'hydratation sont exothermiques. Les adjuvants et la température du milieu influent sur l'étalement de la période dormante. En effet, les études sont limitées en ce qui concerne le rôle des plastifiants et superplastifiants dans l'hydratation du C3S et C3A, c'est pour cette raison qu'on va utiliser deux types de béton, un béton adjuvanté et l'autre non adjuvanté.

La température ambiante joue d'une part un rôle thermodynamique dans l'hydratation du ciment du fait qu'elle change la nature et la stabilité des hydrates et d'autre part un rôle cinétique puisqu'elle retarde ou accélère les réactions chimiques entre l'eau et les composants anhydres.

Pour suivre l'évolution des caractéristiques du béton au jeune âge, la notion du degré d'hydratation est très importante et utile. En effet, elle permet d'intégrer la notion de température à la notion du temps pour déduire une base commune à caractéristiques différentes comme (retrait, résistance à la compression, à la traction par flexion et le module d'élasticité...).

L'hydratation d'une pate de ciment est un processus thermo-activé qui peut être pris par une loi de type Arrhenius [8]. L'évolution du degré d'hydratation peut être décrite par la relation suivante : [6]

$$
\dot{\xi}=\check{A}(\xi) \exp \left(-\frac{E_{a}}{R T}\right)(1) \text { Où }
$$

$\xi$ est le degré d'hydratation,

$\dot{\bar{A}}(\xi)$ désigne l'affinité chimique,

Ea est l'énergie d'activation [J.mol-1],

$\mathrm{R}$ désigne la constante des gaz parfaits [8.314 J.mol-1.K1]

T est la température en Kelvin.

L'affinité chimique normalisée peut être obtenue expérimentalement par un essai adiabatique ou semi adiabatique. Le calorimètre quasi adiabatique est une des méthodes utilisées pour mesurer l'évolution de la température d'une éprouvette en béton pendant la réaction d'hydratation du ciment [9].

L'évolution de la température adiabatique est déduite de l'équation de conservation de l'énergie en supposant que la température de l'éprouvette est uniforme. Dans le calorimètre $\mathrm{QAB}$, une partie de la chaleur libérée due à l'hydratation du ciment augmente la température de l'échantillon tandis qu'une autre partie augmente la température du calorimètre, et le reste se décharge vers l'extérieur. Pour le calorimètre, ces effets sont exprimés comme suit:

$$
\begin{aligned}
& \mathrm{q}(\mathrm{t})=\mathrm{C}_{\text {béton }} \times\left(\mathrm{T}_{\text {adia }}(\mathrm{t})-\mathrm{T}_{\text {béton }}(0)\right)=\mathrm{C}_{\text {tot }} \times \\
& (\theta(\mathrm{t})-\theta(0))+\int_{0}^{\mathrm{t}}(\mathrm{a}+\mathrm{b} \theta(\mathrm{u})) \theta(\mathrm{u}) \mathrm{du}
\end{aligned}
$$

Avec :

Cbéton: Capacité calorifique du béton seul $\left[\mathrm{J} /{ }^{\circ} \mathrm{C}\right]$.

Ctot: Capacité calorifique totale (béton+éprouvette+calorimètre)

$\mathrm{a}[\mathrm{J} / \mathrm{h}]$ et $\mathrm{b}\left[\frac{\mathrm{J}}{\mathrm{h}} /{ }^{\circ} \mathrm{C}\right]$ : Coefficients de déperdition du calorimètre $=$ respectivement 69.7 et 0.113

$\mathrm{t}$ [heure]: Temps de l'essai QAB

$\theta[\mathrm{t}]=$ Tbéton - Text: Echauffement

Une fois la capacité calorifique du béton est connue, on peut calculer l'augmentation de la température dans l'éprouvette placée dans des conditions adiabatiques et on aura :

$$
\mathrm{T}_{\text {adia }}(\mathrm{t})=\mathrm{T}_{\text {béton }}(0)+\frac{\mathrm{q}(\mathrm{t})}{\mathrm{C}_{\text {béton }}}
$$

L'évolution du degré d'hydratation en fonction du temps [7] peut être déterminée par la relation suivante :

$$
\begin{aligned}
& \xi(\mathrm{t})=\xi \infty \frac{\mathrm{T}_{\mathrm{adia}}(\mathrm{t})-\mathrm{T}_{\mathrm{adia}}(0)}{\mathrm{T}_{\infty}^{\text {adia }}-\mathrm{T}_{0}^{\text {adia }}}(4) \quad \text { avec } \\
& \xi \infty=1-\exp \left(-3.25 \times \frac{\mathrm{E}}{\mathrm{C}}\right)
\end{aligned}
$$

\section{Résultats et interprétation :}

\section{Calcul du degré d'hydratation}

Les capacités calorifiques des matériaux et du calorimètre sont données dans le tableau 3[9].

Tableau 4 : Capacité calorifique des matériaux

\begin{tabular}{|l|c|}
\hline \multicolumn{1}{|c|}{ Matériaux } & capacité calorifique $/ /{ }^{\circ} \mathrm{C}$ \\
\hline ciment & 836 \\
\hline gravier 4/8 & 730 \\
\hline gravier 8/16 & 730 \\
\hline sable 0/4 & 835 \\
\hline filler & 840 \\
\hline eau & 4190 \\
\hline adjuvant & 3800 \\
\hline \multicolumn{1}{|c|}{ Matériels } & capacité calorifique $/ /{ }^{\circ} \mathrm{C}$ \\
\hline Moule & 500 \\
\hline Calorimètre & 364 \\
\hline
\end{tabular}


Calcul pour le BAP:

$\mathrm{C}_{\text {béton }}=\left(\mathrm{m}_{\mathrm{c}} \times \mathrm{cc}_{\mathrm{c}}\right)+\left(\mathrm{m}_{\mathrm{g} 1} \times \mathrm{cc}_{\mathrm{g} 1}\right)+\left(\mathrm{m}_{\mathrm{g} 2} \times \mathrm{cc}_{\mathrm{g} 2}\right)+$ $\left(\mathrm{m}_{\mathrm{s}} \times \mathrm{cc}_{\mathrm{s}}\right)+\left(\mathrm{m}_{\mathrm{f}} \times \mathrm{cc}_{\mathrm{f}}\right)+\left(\mathrm{m}_{\mathrm{e}} \times \mathrm{cc}_{\mathrm{e}}\right)+\left(\mathrm{m}_{\mathrm{ad}} \times\right.$ $\left.\mathrm{cc}_{\mathrm{ad}}\right)+\left(\mathrm{m}_{\mathrm{ep}} \times \mathrm{cc}_{\mathrm{ep}}\right)=1094.23 \mathrm{~J} /{ }^{\circ} \mathrm{C}$

$\mathrm{C}_{\text {total BAP }}=\mathrm{C}_{\text {béton }}+\mathrm{CC}_{\text {calorimétre }}=1458.23 \mathrm{~J} /{ }^{\circ} \mathrm{C}$

$\mathrm{C}_{\text {total BV }}=\mathrm{C}_{\text {béton }}+\mathrm{CC}_{\text {calorimétre }}=1397.41 \mathrm{~J} /{ }^{\circ} \mathrm{C}$

L'essai du Calorimètre consiste à mesurer l'évolution de la température d'une éprouvette placée dans une enceinte hermétique et isolée. La figure 4 (courbe en rouge) donne l'enregistrement de la température de l'éprouvette en béton BAP.

Le calcul de la température adiabatique s'effectue selon les équations (2) et (3). La figure 4 (courbe en vert) donne les évolutions de la température adiabatique pour le BAP.

Le calcul du degré d'hydratation s'effectue selon l'équation (4) avec $\xi \infty=1-\operatorname{xp}\left(-3.25 \times \frac{\mathrm{E}}{\mathrm{C}}\right)=0.835$ pour le BAP et 0.803 pour le béton vibré.

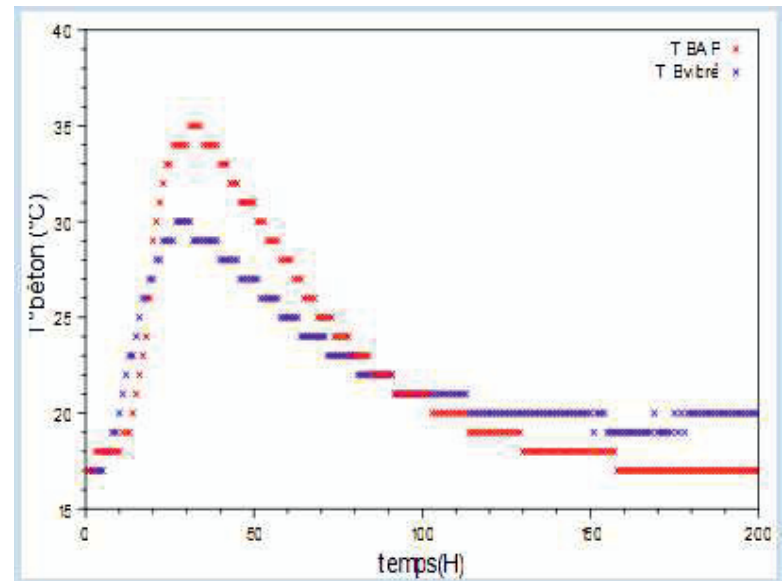

Fig 2 : Evolution de la température du BAP(en rouge) et du béton vibré (en bleu)

La courbe 2 montre l'évolution de la température au cœur de l'éprouvette du béton à l'intérieur du calorimètre de Langavant. Le béton autoplçant présente une température plus élevée, donc une hydratation plus accélérées et cela peut être expliqué par l'effet de l'adjuvant d'une part et d'autre part l'augmentation de la quantité de la pate de liant par l'ajout du filler[13]. La température maximale pour le BAP atteint les $35^{\circ} \mathrm{C}$ après 31 heures et pour le béton vibré non adjuvanté atteint les $30^{\circ} \mathrm{C}$ après 28 heures.

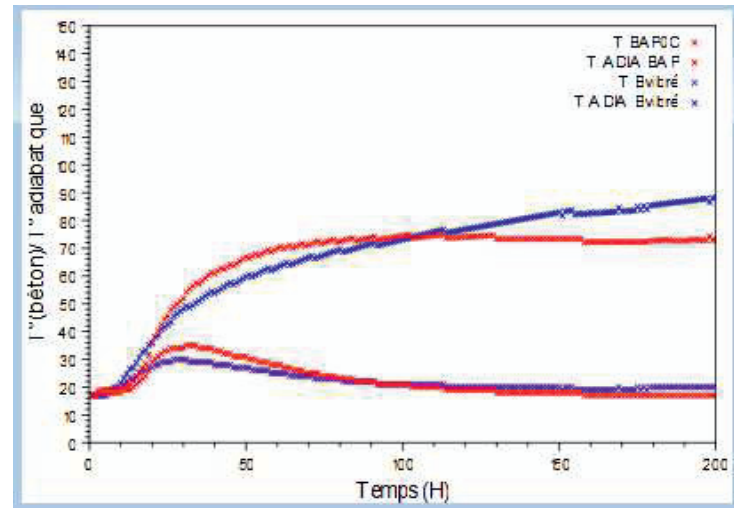

Fig 3 : Evolution de la température adiabatique du BAP(rouge) et Bvibré(Bleu)
La courbe 3 présente l'évolution de la température du béton pour les deux échantillons testés et montre la progression de la température adiabatique calculée à partir de la température du béton en se basant sur l'étude théorique présenté ci-dessus. La température adiabatique est fonction de la température du béton d'une part et d'autre part en fonction de la température ambiante de référence, de la capacité calorifique des constituants du béton et du calorimètre ainsi de la quantité de chaleur dégagées. Ce qu'on peut conclure, on croit que la stabilité adiabatique aperçue pour le béton autoplaçant à partir de 50 heures n'est pas trouvée pour le béton non adjuvanté vibré.

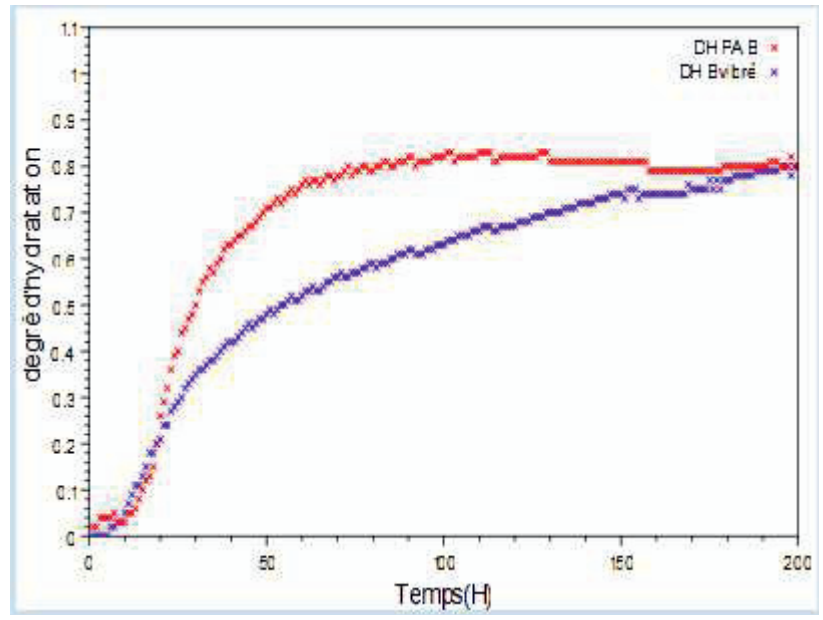

Fig 4 : Evolution du degré d'hydratation en fonction du temps pour le BAP (rouge) et Bvibré (bleu)

La courbe 4 présente la variation du degré d'hydratation en fonction du temps pour les deux échantillons testés. En effet, pour le béton autoplaçant, le degré d'hydratation se stabilise à une valeur de 0.83 notamment supérieure à celle du Bv de l'ordre 0.803 calculé suivant l'exemple ci-dessus. En outre le degré d'hydratation dépend de la température du béton, de la capacité calorifique des constituants et de la température adiabatique calculée ci-dessus. La période critique que montre cette figure est l'intervalle du temps entre 20 et 150 heures où la différence de l'activation de la réaction d'hydratation est importante. Cette différence s'explique $\mathrm{du}$ fait que la contribution de l'influence des adjuvants et des ajouts de calcaire commence à partir de cet instant. Cette contribution n'est très significative dans l'intervalle du temps $[0,20 \mathrm{~h}]$ où les deux courbes sont presque superposées.

\section{Résistances mécaniques}

La relation qui existe entre la résistance mécanique du béton au jeune âge et le degré d'hydratation d'une part et l'indépendance de cette relation avec toute l'histoire thermique suivie par le béton d'autre part peut être validée dans cette partie en se basant sur les essais mécaniques notamment de compression et les essais calorimétriques semi adiabatique de Langavant.

L'évolution des propriétés mécaniques du béton au jeune âge reste importante dans la mesure ou ces évolutions 
conditionnent le comportement mécanique du matériau aux différentes sollicitations et déformations qui sont d'origine thermique et chimique. De plus, ces évolutions peuvent nous prédire sur l'apparition des fissures. La courbe ci-dessous présente les résultats des essais de compression axiale sur des éprouvettes cylindriques $11 \times 22$ conservées à $20^{\circ} \mathrm{C}$ et $98 \%$ d'humidité selon les recommandations de RILEM.

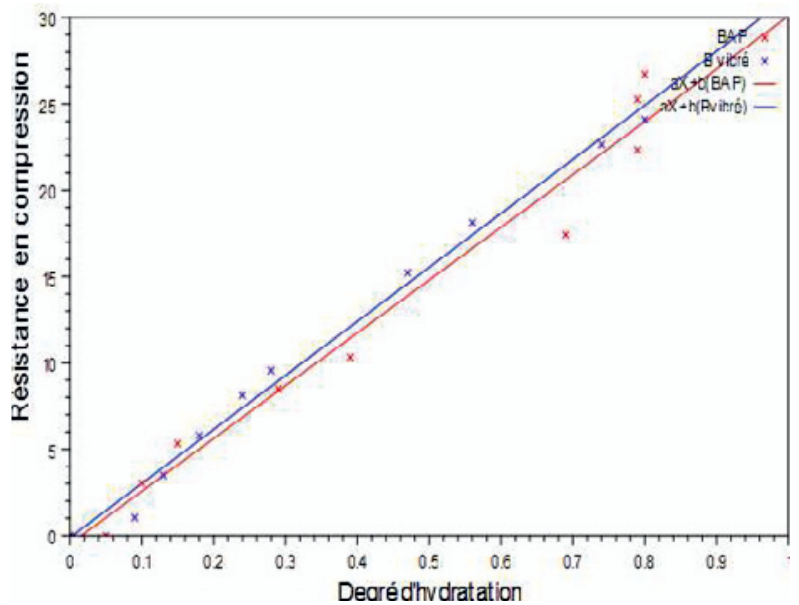

Fig 5 : Evolution de la résistance à la compression en fonction du degré d'hydratation pour le BAP (rouge) et Bvibré (bleu)

Dans cette courbe on confirme la linéarité des résultats de la résistance en compression en fonction du degré d'hydratation des deux bétons. Cette confirmation passe essentiellement par la détermination des paramètres des lois de De Schutter décrivant l'évolution de ces caractéristiques en fonction du degré d'hydratation par la relation suivante :

$B(\xi)=B_{\infty}\left(\frac{\xi-\xi_{0}}{\xi_{\infty}-\xi_{0}}\right)^{a}$ où $\mathrm{B}$ est la variable mécanique.

\section{Conclusion}

La prédiction et en précision de l'évolution de la température dans les ouvrages massives peut être établie suite à aux mesures calorimétrique. La nature de la réaction exothermique du ciment et l'eau peut induire des déformations (dilatation et contraction) qui peuvent causer la fissuration. L'ajout du filaire et des adjuvants peuvent modifier la cinétique de la réaction d'hydratation. Ces facteurs peuvent accélérer la réaction de l'hydratation et par conséquent peuvent induire des contraintes de contraction qui peuvent donner des fissures de retrait En perspective de ce travail, il sera intéressant d'étudier d'autres caractéristiques mécaniques en fonction du degré d'hydratation, l'énergie de fissuration et le comportement du béton au jeune âge.

\section{Références}

[1] ROLS.S, Ambroise J et Péra J, "Effects of different viscosity agents on the properties of self-compacting concrete 》. cement and concrete research,29:461-465 1998 .

[2] De Schutter G. « Guidelines for testing fresh self compacting concrete». European Research Project 2005.
[3] De Schutter G, Tearwe L." self compacting concrete 》. SSC proceeding of the $5^{\text {th }}$ international Rilem symposium RILEM 2007

[4] AFGC. Association Française de génie civil Recommandations provisoires Paris Juillet 2002

[6] F.Ulm., O.Coussy, " coupling in early-age concrete:from material modelling to structural design, International journal of solids and structures 35(31-32) pp 4295-4311

[7] V.Waller 00, " Relation entre composition des bétons, exothermie en cours de prise et résistance en compression, collection études et recherches des laboratoires des ponts et chaussées, série ouvrages d'art:OA35-LCPC,Parie in French

[8] Regourd et Gauthier 1980, "Behavior of cement under accelerated hardening , Annales de l'ITBTP 179,65-96, Paris, France

[9] C. Boulay,J.M. Torrenti, J.L. Andre,R. Saintilan, " Quasi-adiabatic calorimetry for concretes :Influential factors», Bulletin des laboratoires des ponts et chaussées 278 (2010) pp 19-36

[10] Regourd M,Thomassin JH,Baillif P et Touray JC 1980 «Study of the early hydration of Ca3SiO5 by X-ray photoelectron spectrometry to appear in cem.» concr Res volu10 223-230

[11] Mondal P, Jeffery JW 1975«the crystalstructure of tricalcium aluminate»acta cryst B31,689

[12] Barret P, "les mécaniques d'hydratation et de durcissement des ciments "L'industrie nationale $N^{\circ} 1,3,41$.

[13] Gargouri A, Daoud A,Kallel A et Loulizi A, les journées nationales de bétons JNB'13 «Valorisation des granulats de caoutchouc dans un béton autoplaçant; Etude de la cinétique de la réaction d'hydratation»18-19 mai 2013 .Hammamat.Tunisie. 